\title{
Sentinel Lymph Node Biopsy May Prevent Unnecessary Axillary Dissection in Patients with Inflammatory Breast Cancer Who Respond to Systemic Treatment
}

\author{
Hasan Karanlik ${ }^{a}$ Neslihan Cabioglu ${ }^{b}$ Adela Luciana Oprea ${ }^{c} \quad$ Ilker Ozgur ${ }^{b}$ \\ Naziye $A^{d}{ }^{d}$ Adnan Aydiner ${ }^{d} \quad$ Semen Onder ${ }^{e} \quad$ Süleyman Bademler ${ }^{a}$ \\ Bahadir M. Gulluoglu ${ }^{f}$

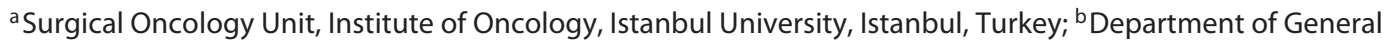 \\ Surgery, Istanbul Faculty of Medicine, Istanbul University, Istanbul, Turkey; ${ }^{\mathrm{C}}$ Department of Gynecology, Targu \\ Mures University of Medicine, Pharmacy, Science and Technology, Targu Mures, Romania; ${ }^{\mathrm{d} D e p a r t m e n t}$ of Medical \\ Oncology, Institute of Oncology, Istanbul University, Istanbul, Turkey; ${ }^{e}$ Department of Pathology, Istanbul Faculty of \\ Medicine, Istanbul University, Istanbul, Turkey; ${ }^{f}$ Breast and Endocrine Surgery Unit, Department of General Surgery, \\ Marmara University School of Medicine, Istanbul, Turkey
}

\section{Keywords \\ Sentinel lymph node biopsy · Inflammatory breast cancer . Neoadjuvant systemic treatment $\cdot$ HER2-positive breast cancer - Triple-negative breast cancer}

\begin{abstract}
Background and Objectives: Inflammatory breast cancer (IBC) is a rare and aggressive breast cancer treated up-front with systemic treatment. Both breast-conserving surgery and sentinel lymph node biopsy (SLNB) are controversial issues in the management of IBC. In this study, we aimed to assess the feasibility of SLNB in pathologically proven nodepositive IBC patients. Methods: All patients with a histopathological diagnosis of IBC and biopsy-proven metastatic axillary lymph nodes underwent systemic treatment. Patients with a complete clinical response in the axilla who underwent SLNB followed by standard axillary dissection were analyzed. Results: The study consisted of 25 female patients. The identification rate (IR) and the false negativity rate (FNR) were $17 / 25$ and $2 / 10$, respectively. Overall, 9/25 and $7 / 25$ of patients had a complete pathological response $(p C R)$ in the breast and axilla after systemic treatment, respectively. Although the pCR in the axilla was $2 / 4$ in nonluminal HER2-positive patients, the highest IR $4 / 4$ and the
\end{abstract}

lowest FNR 0/2 were determined in these patients. In triplenegative patients, however, the IR was $2 / 4$ and the FNR was found to be $0 / 2$. Conclusions: SLNB may be considered in selected axilla-downstaged IBC patients including patients with a pCR with HER2-positive and triple-negative tumors. Axillary dissection may be, therefore, omitted in those with negative SLNs.

(c) 2020 S. Karger AG, Basel

\section{Introduction}

Inflammatory breast cancer (IBC) is a rare but aggressive type of malignant breast disease. It is regarded as advanced cancer due to its poor prognosis despite multimodality treatment. IBC presents with extensive involvement of the breast as well as a high axillary metastatic burden. Its clinical presentation therefore excludes R0 surgical resection in most cases. This is the reason that systemic up-front treatment is recommended even in the nonmetastatic stage, and surgical eradication of the disease is only attempted if the patient responds well to this treatment. Total mastectomy and full axillary clearance are accepted as the surgical treatment modality for IBC patients $[1,2]$. However, due to the introduction of more 
effective systemic treatments, as observed in noninflammatory advanced breast cancer, recently, more patients have had clinical downstaging both in the breast and axilla. Recent studies show that systemic treatment provides an overall pathological complete response (pCR) in 30$45 \%$ of IBC patients [3-6]. The response rate varies according to the molecular subtype of cancer. In HER2positive IBC patients, pCR rates in the breast and axilla were found to be 43 and $56 \%$, respectively, after up-front systemic treatment [6]. These findings nudged surgeons to question the necessity of mastectomy and axillary clearance in these patients who respond well to systemic treatment $[7,8]$.

Sentinel lymph node biopsy (SLNB) is an accepted method for assessing the ipsilateral axilla in breast cancer patients who have clinically responded to systemic treatment [9]. However, SLNB after systemic treatment in IBC patients remains controversial. Until now, only a few studies have assessed the identification rate (IR) and false-negativity rate (FNR) of SLNB in IBC patients having clinical axillary downstaging after receiving systemic treatment [10-12]. In these studies, IRs were low and FNRs were high. However, SLNB is a valid alternative for the subgroup of patients expected to respond well to systemic treatment. Until now, only 1 study provided results for each cancer subtype. However, this study had a limited sample size and a very low IR, so no relevant conclusion was drawn for the impact of subtyping [12].

This study aimed to assess the feasibility of SLNB in a larger group of IBC patients who responded well to systemic treatment in the axilla. The IR and FNR calculations were separately made in the groups with different cancer subtypes. Correlation between SLNB accuracy and residual cancer burden in the breast was also assessed.

\section{Material and Methods}

\section{Design}

This study was planned as a retrospective registry trial requiring data retrieval from a single tertiary breast health care institution. All study variables were taken from a prospectively kept clinical database. The database was screened for patients with IBC admitted to the institution from 2010 until the study date. All patients with clinically diagnosed IBC who underwent SLNB followed by consecutive axillary dissection after systemic treatment were included.

\section{Inclusion and Exclusion Criteria}

Patients who were clinically diagnosed as having IBC with no distant metastasis, had any axillary status at diagnosis but were clinically node-negative after systemic treatment, had undergone complete immunohistochemistry (IHC) subtyping of the primary tumor (in an up-front core biopsy if pCR was achieved), and completion axillary dissection were included in the study.

On the other hand, patients with distant metastases and/or extra-axillary locoregional lymph node involvement, who did not receive systemic treatment nor undergo SLNB, who underwent SLNB but no further completion axillary dissection, and with missing data regarding their response to systemic treatment and/or features of the primary tumor pathology were excluded from the analysis.

\section{Outcomes}

Primary study outcomes were IR and FNR overall in all patients. We also calculated the overall accuracy $(\mathrm{OA})$ as a secondary outcome. Furthermore, we reported the IR, FNR, and OA rate according to each molecular cancer subtype, each level of systemic treatment response, and the number of retrieved SLNs.

\section{Variables}

Other variables collected from the database included a patient's age, gender, axillary $(\mathrm{N})$ and tumor size $(\mathrm{T})$ stages before systemic treatment, the type of systemic treatment (regimen and course), the completion rate of systemic treatment, the type of breast surgery, the number of retrieved SLNs, the number and size of SLN metastases (if any), the number of axillary lymph nodes retrieved by completion axillary dissection (non-SLN), and the number of metastatic non-SLNs (if any).

\section{Assessment of Patients for Staging before and after Systemic \\ Treatment}

All patients underwent a physical exam of the breast and axilla after tissue diagnosis by core biopsy. Bilateral mammography, breast ultrasound (US), and breast MRI were the standard tools used to assess the locoregional extension of the disease. Distant disease was ruled out by PET-CT imaging in all patients. Axillary involvement of each patient was determined either by a combination of clinical examination, axilla US, PET-CT imaging, or USguided fine-needle aspiration biopsy (FNAB) in patients with clinically suspicious nodes. If there was an accompanying tumor mass in the breast, the size of the tumor was measured by US.

After completion of the systemic treatment, all patients had breast and axilla US, breast MRI, and a physical exam to assess their clinical response to systemic treatment. If there was no palpable and ultrasonographically suspicious lymph node appearance in the ipsilateral axilla, the patient was regarded as clinical ynN0, and was thus eligible for SLNB.

\section{Systemic Treatment}

All patients were examined and assessed by a multidisciplinary team consisting of a breast surgeon, radiologist, and medical oncologist. Decisions about systemic treatment, the regimen, and the duration were decided by the team. Patients received 4 cycles of adriamycine (A) with cyclophosphamide (C), followed by 4 cycles of taxane (T) or 6 cycles of TAC. Anti-HER2 drugs were included for patients with HER2 overexpression.

\section{Surgery of the Breast and Axilla}

SLNB and axillary lymph node dissection have been performed in every patient who underwent primary chemotherapy with the diagnosis of locally advanced breast cancer with the consent of the patient as an institutional clinical decision since 2010. However, there is no SLNB indication for IBC patients in internationally accepted guidelines.

Every patient underwent a total mastectomy with SLNB followed by standard level I-II axillary dissection according to standard surgical principles and techniques. Combined tracers (radioisotope + blue dye), or blue dye only, were used to trace the SLN in the ipsilateral axilla. Technetium-99 sulfur colloid was the radioisotope used. This was injected the day before surgery; blue dye is injected to the periareolar area during surgery. After 5 min of 
massage, any blue-stained and/or hot nodes were examined through an incision over the axilla or a mastectomy incision. Further axillary dissection was done to clear all lymph nodes at levels I and II. Following this stage, patients underwent standard breast surgery.

\section{Pathological Assessment and Molecular Subtyping}

Breast (The Primary Tumor)

Paraffin-embedded breast tissue obtained from core biopsy was microcut and stained with H\&E. The cancer diagnosis was confirmed by its histologic morphology. Epithelial type and grade of cancer in each patient were given. Then, further IHC staining for estrogen receptor (ER), progesterone receptor (PR), HER2, and Ki67 were done. The expression level of each marker was given accordingly.

A breast excision specimen obtained from definitive surgery after systemic treatment was also assessed. Paraffin-embedded tissue was cut into 5-mm-thick sections and stained with H\&E. Any residual cancer burden was reported accordingly after histological microscopic assessment. If there were any cancer cells remaining, the size and invasive pattern were reported.

Axilla (Lymph Nodes)

After systemic treatment, either SLNs or lymph nodes cleared during axillary dissection were embedded in paraffin. The block of each lymph node was cut into 2-mm-thick sections and stained with H\&E. Each slide was histopathologically reviewed under a light microscope for the presence of any metastatic cancer clusters. If there was any, metastasis was classified according to its size as macrometastasis, micrometastasis, or isolated tumor cells (ITC).

Patients were grouped into molecular subtypes as luminal $\mathrm{A}$, luminal B, luminal HER2-positive, nonluminal HER2-positive, and triple-negative according to the IHC results obtained from a Tru-Cut biopsy specimen after systemic treatment. In patients with $\mathrm{PCR}$, the IHC findings were regarded as final to categorize patients into subgroups.

Classification of the Response to the Treatment in the Breast

Responses were classified into 3 groups: complete (pCR), partial (pPR), and none (pNR) $[10,13]$. pCR was defined as the absence of all cancer cells in the breast (either the invasive or in situ form). pNR was defined as a sum of stable and progressive diseases. PPR was defined as all cases that did not fit into either the $\mathrm{pCR}$ or the pNR group. Therefore, other response levels less than partial response were regarded as "non-response".

\section{IHC Staining and Assessment (Criteria for Positivity/}

Negativity)

Patients were regarded as ER-positive and/or PR-positive if at least $1 \%$ expression of each was found. Patients were regarded as HER2-positive if their tumor expressed "+++ staining" on IHC or was found to be positive after any type of in situ hybridization technique.

For molecular subtyping, the tumor was regarded as luminal A if ER and PR were both highly expressed ( $>80 \%$ each) in cases negative for HER2 with Ki67 expression $<15 \%$. Luminal B was designated if PR expression was low $(<20 \%)$ in cases negative for HER2 with Ki67 expression $\geq 15 \%$. Luminal HER2-positive was designated if HER2 was positive with ER and/or PR expression $>1 \%$ (regardless of Ki67 expression). Nonluminal HER2-positive was designated if HER2 was positive with no ER and/or PR expression $<1 \%$ (regardless of Ki67 expression), and triple negativity was designated if any expressions were negative regardless of Ki67 expression.

\section{Statistics}

IR, FNR, and OA rate were calculated and results for the total cohort were given to reflect the success of SLNB in these selected patients. Rates were also given for each cancer molecular subtype group as a descriptive analysis, but with no statistical comparison as the sample size in each group was too small or there was none. This was also done for groups defined according to the level of response to systemic treatment. Finally, rates were given for patients with different numbers of SLNs retrieved, i.e., $\geq 3$ versus $<3$ SLNs.

By definition, finding any metastatic deposit including ITC in any SLN meant this was regarded as a "positive SLN". However, only macrometastatic and micrometastatic tumor involvement in the remaining lymph nodes (non-SLNs) were regarded as "positive axilla" excluding ITC. The IR was calculated by dividing the number of patients in whom at least 1 SLN was found and retrieved into the number of all patients who underwent SLNB. The FNR was calculated by dividing the number of patients whose SLN was negative but in whom the non-SLN was found to be metastatic after completing the axillary dissection (i.e., false-negative) into the number of all patients whose axilla was found to have metastasis (including those in whom only the SLN was metastatic, i.e., false-negative and truepositive cases). The OA rate was calculated by dividing the number of patients with positive SLNs and those with overall negative axillae (at both SLNs and lymph nodes at the remaining axilla, i.e., truepositive + true-negative cases) into the total number of patients.

\section{Results}

\section{Cohort Details}

Twenty-five patients who met the inclusion criteria were included in the study. In all patients, cancer was unilateral. They were operated at the Breast Surgery Unit at the Istanbul University Institute of Oncology, between February 2010 and June 2018. All patients were female. The median age of the cohort was 47 (range 26-74) years. Most patients (16/25) had N1 disease at the initial presentation before any treatment. There was a minimum of 1 cancer mass accompanying inflammatory changes in the breast in 16 patients. The median size of the accompanying cancer was $50 \mathrm{~mm}$ (range 3-160 mm). Luminal HER2-positive $(n=10)$ was the most common molecular cancer subtype. All patients completed their planned systemic treatment. All patients underwent total mastectomy. Using only blue dye $(n=14)$ was the most common method for identifying SLNs. Details of patients' demographic and clinical characteristics are given in Table 1.

\section{Pathological Response in the Breast to Systemic \\ Treatment}

Nine (36\%) patients had pCR and 16 (64\%) had pPR in the breast. None of the patients had pNR. None of the patients with luminal A cancer had pCR. Among 10 patients with luminal B cancer, 2 had pCR. Among 5 patients with luminal HER2-positive cancer, 2 had pCR. All 4 patients with nonluminal HER2-positive cancer had pCR. Among 4 patients with triple-negative cancer, 1 had pCR (Table 2). 
Table 1. Demographic and clinical characteristics of the study cohort

\begin{tabular}{|c|c|}
\hline Median age, years (range) & $46(26-74)$ \\
\hline \multicolumn{2}{|l|}{ Gender } \\
\hline Female & $25(100)$ \\
\hline Male & $0(0)$ \\
\hline \multicolumn{2}{|l|}{ Menopausal status } \\
\hline Premenopausal & $17(68)$ \\
\hline Perimenopausal & $0(0)$ \\
\hline Postmenopausal & $8(32)$ \\
\hline \multicolumn{2}{|l|}{ PreNST axillary stage } \\
\hline N0 & $2(8)^{\mathrm{b}}$ \\
\hline N1 & $16(64)$ \\
\hline $\mathrm{N} 2$ & $6(24)$ \\
\hline N3 & $1(4)$ \\
\hline \multicolumn{2}{|l|}{ Tumor histology } \\
\hline Ductal & $19(76)$ \\
\hline Lobular & $4(16)$ \\
\hline Mixed & $2(8)$ \\
\hline \multicolumn{2}{|l|}{ Tumor subtype } \\
\hline Luminal A & $2(8)$ \\
\hline Luminal B & $10(40)$ \\
\hline Luminal HER2 & $5(20)$ \\
\hline Nonluminal HER2 & $4(16)$ \\
\hline Triple-egative & $4(16)$ \\
\hline Median pre-NST tumor size ${ }^{\mathrm{a}}, \mathrm{mm}$ (range) & $45(20-200)$ \\
\hline \multicolumn{2}{|l|}{ NST regimen $^{c}$} \\
\hline $4 \times \mathrm{AC}+4 \times \mathrm{T}$ & $21(84)$ \\
\hline $6 \times \mathrm{TAC}$ & $4(16)$ \\
\hline \multicolumn{2}{|l|}{ Planned NST } \\
\hline Completed & $100(0)$ \\
\hline Not completed & $0(0)$ \\
\hline \multicolumn{2}{|l|}{ Type of breast surgery } \\
\hline Mastectomy & $100(100)$ \\
\hline Wide local excision & $0(0)$ \\
\hline \multicolumn{2}{|l|}{ Agents for SLNB } \\
\hline Blue dye only & $14(56)$ \\
\hline Blue dye + radioisotope & $11(44)$ \\
\hline \multicolumn{2}{|c|}{ 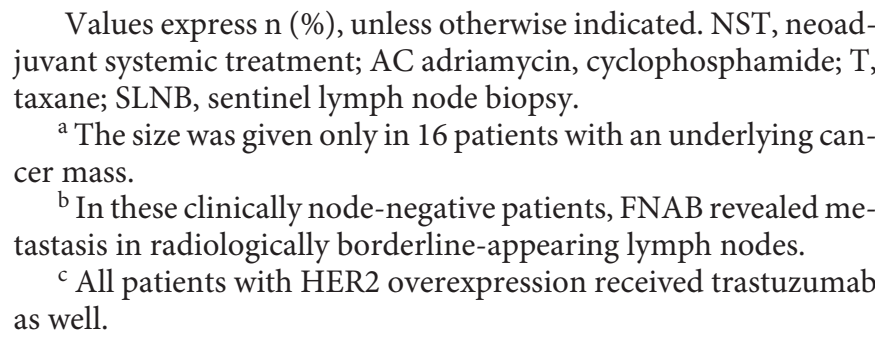 } \\
\hline
\end{tabular}

\section{Pathological Response in the Axilla to Systemic \\ Treatment}

Seven patients were found to have pCR in their axilla after neoadjuvant systemic therapy (NST). The axilla was found to be downstaged to $\mathrm{pN} 0$ in $0 / 2,2 / 10,2 / 5,2 / 4$, and $1 / 4$ of patients with luminal A, luminal B, luminal HER2positive, nonluminal HER2-positive, and triple-negative cancers, respectively. All patients with pCR in the axilla were also found to have PCR in their breasts.

\section{Identification Rate}

In our study cohort, at least 1 SLN was found and excised for pathological assessment in 17 patients, and the IR was calculated as $68 \%$. The median number of SLNs retrieved was $1(1-3)$ in those patients with a successful mapping. The IR was $1 / 2,7 / 10,3 / 5,4 / 4$, and $2 / 4$ in patients with luminal A, luminal B, luminal HER2-positive, nonluminal HER2-positive, and triple-negative cancer, respectively (Table 3 ). Furthermore, the IR was $9 / 9$ and $8 / 16$ in patients with pCR and PPR in the breast, respectively (Table 4).

\section{False Negativity Rate}

Among those in whom SLNB was successful, 8 patients had metastasis in at least 1 of their SLNs, 7 with macrometastasis and 1 with micrometastasis. In the patients with successful mapping, the median total number of axillary lymph nodes retrieved by SLNB and following axillary clearance was 9 (4-23). Axillary lymph node dissection yielded $<10$ lymph nodes in 5 patients. In 2 patients with nonmetastatic SLN, the remaining axillary lymph nodes were found to be metastatic after further dissection. Therefore, the FNR of the cohort was found to be $2 / 10$. The SLN was metastatic in 1 successfully mapped luminal A case; no relevant FNR could be calculated due to there being no cases with a negative SLN in this subgroup.

Among 7 successfully mapped luminal B cases, 2 were found to have negative SLNs and nonsentinel axillary lymph nodes. In 3 patients, both the SLN and the remaining axilla were positive. One patient had metastatic SLN but no positive lymph nodes in the remaining axilla. One patient had a negative SLN, but the remaining axillary lymph nodes were metastatic. Therefore, the FNR was calculated as $1 / 5$ in patients with luminal $\mathrm{B}$ cancer.

Among 3 successfully mapped luminal HER2-positive cases, both SLNs and remaining axillary lymph nodes were negative in 2 patients. One patient had a negative SLN but her remaining axillary lymph nodes were metastatic. Therefore, the FNR was calculated as $1 / 1$ in patients with luminal HER2-positive cancer.

Among 4 successfully mapped nonluminal HER2positive cases, both SLNs and remaining axillary lymph nodes were negative in 2 patients. In 2 patients, both the SLNs (1 with micrometastasis) and the remaining axilla were positive. Therefore, the FNR was calculated as $0 / 2$ in patients with nonluminal HER2-positive cancer.

Among 2 successfully mapped triple-negative cases, in 1 patient, both SLNs and remaining axillary lymph nodes were negative. In the other patient, both SLNs and the remaining axillary lymph nodes were metastatic. Therefore, the FNR was calculated as $0 / 2$ in patients with triplenegative cancer versus an FNR of $0 / 9$ and $2 / 8$ in patients with pCR and pPR, respectively (Table 4). 
Table 2. Pathological response in the breast according to cancer subtypes

\begin{tabular}{lllllll}
\hline & Luminal A & Luminal B & Luminal HER2 & Nonluminal HER2 & Triple-negative & Overall \\
\hline pCR & $0 / 2(20)$ & $2 / 10(20)$ & $2 / 5(40)$ & $4 / 4(100)$ & $1 / 4(25)$ & $9 / 25(36)$ \\
pPR & $2 / 2(80)$ & $8 / 10(80)$ & $3 / 5(60)$ & $0 / 4(0)$ & $3 / 4(75)$ & $16 / 25(64)$ \\
pNR & $0 / 2(0)$ & $0 / 10(0)$ & $0 / 5(0)$ & $0 / 4(0)$ & $0 / 4(0)$ & $0 / 25(0)$ \\
\hline
\end{tabular}

Values express $n /$ total number of cancer subtypes (\%). Pathological response rate: complete (pCR), partial (pPR), none (pNR).

Table 3. Identification rate, false negativity rate, and overall accuracy rate of SLNB according to cancer subtypes in IBC patients

\begin{tabular}{llclllr}
\hline & Luminal A & Luminal B & Luminal HER2 & Nonluminal HER2 & Triple-negative & Overall \\
\hline Identification rate & $1 / 2(50)$ & $7 / 10(70)$ & $3 / 5(60)$ & $4 / 4(100)$ & $2 / 4(50)$ & $17 / 25(68)$ \\
False negative rate & n.a. & $1 / 5(20)$ & $1 / 1(100)$ & $0 / 2(0)$ & $0 / 2(0)$ & $2 / 10(20)$ \\
Overall accuracy rate & $1 / 1(100)$ & $6 / 7(86)$ & $2 / 3(67)$ & $4 / 4(100)$ & $2 / 2(100)$ & $15 / 17(88)$ \\
\hline
\end{tabular}

Values express $n /$ total number of cancer subtypes (\%). ${ }^{*}$ n.a., not applicable.

When stratifying patients according to the number of SLNs retrieved, 3 SLNs were retrieved in 1 patient only, and the remaining 16 patients had $<3$ SLNs. In the patient with 3 SLNs, these were found to be metastatic. Therefore, all false-negative cases were observed in those who had $\leq 2$ SLNs.

\section{OA Rate}

In the study cohort, the OA rate was $15 / 17$. When stratifying patients according to the number of SLNs retrieved, the OA rate was $1 / 1$ and $14 / 16$ in patients in whom $\geq 3$ and $<3$ SLNs were retrieved, respectively. The OA rate was $1 / 1,6 / 7,2 / 3,4 / 4$, and $2 / 2$ in patients with luminal A, luminal B, luminal HER2-positive, nonluminal HER2-positive, and triple-negative cancer, respectively (Table 3 ). The OA rate was $9 / 9$ and $6 / 8$ in patients with $\mathrm{pCR}$ and $\mathrm{pPR}$, respectively (Table 4 ).

\section{Discussion}

In this retrospective study, we found that SLNs could be identified in 17/25 (68\%) of IBC patients who had a clinically complete response in the axilla after systemic treatment, regardless of their subtype. In all patients with nonluminal HER2-positive cancer, at least 1 SLN was retrieved; in those with luminal A and triple-negative cancer, the SLN was successfully mapped in only half of the cases. In all patients who had pCR in the breast, the SLN was found after mapping regardless of their subtype. On
Table 4. Identification rate, false negativity rate, and overall accuracy rate of SLNB according to the level of pathological response in the breast after systemic therapy in IBC patients

\begin{tabular}{llll}
\hline & pCR & pPR & pNR \\
\hline Identification rate & $9 / 9(100)$ & $8 / 16(50)$ & n.a. \\
False negative rate & $0 / 9(0)$ & $2 / 8(25)$ & n.a. \\
Overall accuracy rate & $9 / 9(100)$ & $6 / 8(75)$ & n.a. \\
\hline
\end{tabular}

Values express $n /$ total number of cancer subtypes (\%). n.a., not applicable.

the other hand, the overall FNR was 2/10. In patients with nonluminal HER2 and triple-negative cancer, there were no false-negatives. Also, there was no FNR in patients with pCR in their breast, but an FNR of $2 / 8$ in those with pPR.

Until now, 3 studies have reported the IR and FNR in IBC patients after systemic treatment [10-12]. In general, all of the studies reported discouraging results regarding SLNB attempts in this subset of patients who are believed to have a dismal prognosis overall. In the earliest of these studies, only 8 patients with IBC had SLNB after NST. In this study, in which only blue dye was used for guidance, the IR was $75 \%$. Among the 6 patients with successful identification, 5 were found to have metastatic lymph nodes in the remaining axilla after complete dissection. Among these, 2 patients had negative SLNs. Therefore, the FNR was $40 \%$ [10]. In another study, in which only blue 
dye was used for SLN identification, the IR was reported to be $80 \%$ in 20 IBC patients who were clinically nodenegative after systemic treatment. In this study, the FNR was $18 \%$ among 11 patients in which the SLNB was successful [11]. In both studies, the preoperative node status of the patients was determined clinically and was not confirmed on biopsy. Furthermore, no stratification according to molecular subtypes was done in these studies.

In a recent, prospectively designed study in which SLNB was done in 16 patients by using a dual agent (blue dye and radiotracer), the authors were able to retrieve a minimum 1 SLN in only 4 cases. Therefore, their IR was $25 \%$. All 4 patients with successful mapping had either HER2-positive or triple-negative cancer. Furthermore, $3 / 4$ patients had pCR in the axilla. Therefore, in this particular study, the calculation of the FNR was not relevant due to the low number of samples available for meaningful analysis. Another drawback was the inclusion of patients who remained with abnormal findings in their lymph nodes after systemic treatment, i.e., their response could not be regarded as a convincing indication for SLNB [12]. However, in our study, we included only those cases with a satisfactory response in the axilla as evaluated on US after completing the systemic treatment. This strategy might have resulted in a higher rate of IR overall in our study, especially in those patients with cancer subtypes that respond well to systemic treatment.

With the help of effective neoadjuvant systemic treatment, especially in HER2-positive and triple-negative cancer, the rate of pCR both in the breast and axilla is increasing in IBC patients. The response is far better in triple-negative and HER2-positive cancer [3-6]. The pCR in the axilla was found to be 65 and $38 \%$ in HER2-positive and triple-negative IBC patients, respectively [6]. Therefore, in contrast to recent belief, even in IBC there seems to be a group of patients who can be saved from unnecessary axillary dissection. Our findings have shown that the IR is high in nonluminal HER2-positive cancer patients. We also found no false-negative cases in the subgroup with the highest $\mathrm{pCR}$ rate among all patients. Therefore, IBC patients with a high probability of achieving $\mathrm{pCR}$ would be the best candidates to get the highest benefit from SLNB after NST. From the biological standpoint, this group is more likely to be composed of HER2-positive and triple-negative breast cancer patients due to the likelihood that they will respond to systemic treatment. In our series, the IR and FNR were found to be 9/9 and $0 / 9$ in patients with breast pCR. As expected, the response was highest $(2 / 4$ pCR in the axilla and $4 / 4$ pCR in the breast) in patients with nonluminal HER2-positive cancer; this finding translated into a better IR and FNR in these patients. Although their IR was low, the triple-negative cancer patients expected to give good response to systemic treatment had an FNR of 0/2 as well.
To our knowledge, this is the largest case series reported until now studying the feasibility of SLNB in biopsyproven node-positive IBC patients who had a clinical complete response in the axilla after systemic treatment. Due to our high IR in the small study group, we could calculate the FNR in a large subset of patients in this rare breast cancer patient group. We also compared the IR and FNR in each biological subtype, and according to the level of pathological response in the breast. To keep the missing data at a minimum, all variables were retrieved from a prospectively kept database. Furthermore, all patients were operated on by the same surgeon (H.K.), and this enabled us to keep the standards and homogeneity in surgical techniques.

Nevertheless, there are certain limitations when considering the relevance of our study. Although it presents the largest sample until now, it was still relatively small. This was mainly due to the fact that IBC is a rare condition [2]. This limited our statistical power to make more reliable and relevant conclusions. However, our results can be considered as strong evidence that SLNB is an option in certain conditions in IBC patients after systemic treatment. Another drawback of our study was the heterogeneity of the SLNB technique that we applied in patients. Although using dual tracer maximized the IR and decreased the FNR in patients who received systemic treatment [14-16], we used radiotracer and blue dye as a combined technique in less than half of the cases. In the rest, we relied on 1 blue dye only. Both the nonuniformity of the technique and the substandard method might have contributed to the low IR and high FNR in this study. However, there are also studies that have found identification better with blue dye [17]. According to the Alliance trial [11], performing dual tracer and harvesting 3 lymph nodes during SLNB may increase the IR in conjunction with an FNR decrease. The limited number of SLNs retrieved in our cases can be considered another drawback since the IR and FNR are suboptimal when only 1 or 2 SLNS are retrieved after systemic treatment [14-16]. Furthermore, backup axillary dissection in our study yielded $<10$ lymph nodes in 5 patients, i.e., fewer lymph nodes than required for an adequate axillary dissection to calculate the FNR. One more point which should be kept in consideration is that $2 / 3$ of our patients had N1 disease before undergoing systemic treatment. Our results should be met with caution, especially for those patients with more extensive axillary disease at the first admission.

\section{Conclusions}

As better and more effective systemic treatment modalities have been introduced, more patients achieve pCR after systemic treatment. This is also evident in IBC pa- 
tients. Therefore, improvement in disease response rates to systemic treatment increases the likelihood of performing SLNB with acceptably low FNRs. This study suggests that in selected IBC patients, especially those who achieve pCR in the breast, SLNB may be considered as an option for axillary assessment. Accurate assessment of the axilla on US after systemic treatment may be able to identify patients who will benefit from SLNB and thereby spare some from undergoing more extensive surgery. Patients with triple-negative and HER2-positive IBC cancer may benefit from SLNB after systemic treatment if they achieve a very good clinical response. However, a larger sample size is required to approve the validity of SLNB in these IBC patients before implementating it in current practice.

\section{Statement of Ethics}

This article was written in accordance with the ethics standards of the institutional review board and the 1964 Helsinki Declaration and its later amendments or comparable standards. Ethics Committee approval was from Institutional Review Board of Institute of Oncology, Istanbul University (No. 2015-398). Informed consent was obtained from all individual participants included in the study.

\section{Conflict of Interest Statement}

The authors declare there are no potential conflicts of interest with respect to the research, authorship, and/or publication of this article.

\section{Funding Sources}

There was no funding, sponsor, or financial support.

\section{Author Contributions}

H.K., N.C., I.O., A.A., and B.M.G. Substantial contributions to the conception or design of the work. H.K., A.L.O., I.O., N.A., A.A., S.O., S.B., and B.M.G. Acquisition, analysis, or interpretation of data for the work. H.K., N.C., I.O., A.A., S.O., and B.M.G. Drafting the work or critical revision for important intellectual content. H.K., N.C., A.L.O., I.O., N.A., A.A., S.O., S.B., and B.M.G. Final approval of the version to be published.

\section{Data Availability}

The data that support the findings of this study are available on request from the corresponding author. The data are not publicly available due to privacy or ethical restrictions.

\section{References}

1 Dawood S, Merajver SD, Viens P, Vermeulen $\mathrm{PB}$, Swain SM, Buchholz TA, et al. International expert panel on inflammatory breast cancer: consensus statement for standardized diagnosis and treatment. Ann Oncol. 2011 Mar;22(3):515-23.

2 Menta A, Fouad TM, Lucci A, Le-Petross $\mathrm{H}$, Stauder MC, Woodward WA, et al. Inflammatory Breast Cancer: What to Know About This Unique, Aggressive Breast Cancer. Surg Clin North Am. 2018 Aug;98(4):787-800.

3 Gentile LF, Plitas G, Zabor EC, Stempel M, Morrow M, Barrio AV. Tumor Biology Predicts Pathologic Complete Response to Neoadjuvant Chemotherapy in Patients Presenting with Locally Advanced Breast Cancer. Ann Surg Oncol. 2017 Dec;24(13): 3896-902.

4 Rosso KJ, Tadros AB, Weiss A, Warneke CL, DeSnyder S, Kuerer H, et al. Improved Locoregional Control in a Contemporary Cohort of Nonmetastatic Inflammatory Breast Cancer Patients Undergoing Surgery. Ann Surg Oncol. 2017 Oct;24(10):2981-8.

5 Hieken TJ, Murphy BL, Boughey JC, Degnim AC, Glazebrook KN, Hoskin TL. Influence of Biologic Subtype of Inflammatory Breast Cancer on Response to Neoadjuvant Therapy and Cancer Outcomes. Clin Breast Cancer. 2018 Aug;18(4):e501-6.

6 van Uden DJ, van Maaren MC, Bult P, Strobbe LJ, van der Hoeven JJ, Blanken-Peeters CF, et al. Pathologic complete response and overall survival in breast cancer subtypes in stage III inflammatory breast cancer. Breast Cancer Res Treat. 2019 Jul;176(1):217-26.
7 Chen $\mathrm{H}$, Wu K, Wang M, Wang F, Zhang M, Zhang P. A standard mastectomy should not be the only recommended breast surgical treatment for non-metastatic inflammatory breast cancer: A large population-based study in the Surveillance, Epidemiology, and End Results database 18. Breast. 2017 Oct;35:4854 .

8 Brzezinska M, Williams LJ, Thomas J, Michael Dixon J. Outcomes of patients with inflammatory breast cancer treated by breastconserving surgery. Breast Cancer Res Treat. 2016 Dec;160(3):387-91.

9 Curigliano G, Burstein HJ, Winer EP, Gnant M, Dubsky P, Loibl S, et al.; St. Gallen International Expert Consensus on the Primary Therapy of Early Breast Cancer 2017. De-escalating and escalating treatments for earlystage breast cancer: the St. Gallen International Expert Consensus Conference on the Primary Therapy of Early Breast Cancer 2017. Ann Oncol. 2017 Aug;28(8):1700-12.

10 Stearns V, Ewing CA, Slack R, Penannen MF, Hayes DF, Tsangaris TN. Sentinel lymphadenectomy after neoadjuvant chemotherapy for breast cancer may reliably represent the axilla except for inflammatory breast cancer. Ann Surg Oncol. 2002 Apr;9(3):235-42.

11 Hidar S, Bibi M, Gharbi O, Tebra S, Trabelsi A, Korbi S, et al. Sentinel lymph node biopsy after neoadjuvant chemotherapy in inflammatory breast cancer. Int J Surg. 2009 Jun; 7(3):272-5.

12 DeSnyder SM, Mittendorf EA, Le-Petross C, Krishnamurthy S, Whitman GJ, Ueno NT, et al. Prospective Feasibility Trial of Sentinel
Lymph Node Biopsy in the Setting of Inflammatory Breast Cancer. Clin Breast Cancer. 2018 Feb;18(1):e73-7.

13 Eisenhauer EA, Therasse P, Bogaerts J, Schwartz LH, Sargent D, Ford R, et al. New response evaluation criteria in solid tumours: revised RECIST guideline (version 1.1). Eur J Cancer. 2009 Jan;45(2):228-47.

14 Boughey JC, Suman VJ, Mittendorf EA, Ahrendt GM, Wilke LG, Taback B, et al.; Alliance for Clinical Trials in Oncology. Sentinel lymph node surgery after neoadjuvant chemotherapy in patients with node-positive breast cancer: the ACOSOG Z1071 (Alliance) clinical trial. JAMA. 2013 Oct;310(14):145561.

15 Kuehn T, Bauerfeind I, Fehm T, Fleige B, Hausschild M, Helms G, et al. Sentinellymph-node biopsy in patients with breast cancer before and after neoadjuvant chemotherapy (SENTINA): a prospective, multicentre cohort study. Lancet Oncol. 2013 Jun; 14(7):609-18.

16 Boileau JF, Poirier B, Basik M, Holloway CM, Gaboury L, Sideris L, et al. Sentinel node biopsy after neoadjuvant chemotherapy in biopsy-proven node-positive breast cancer: the SN FNAC study. J Clin Oncol. 2015 Jan;33(3): 258-64.

17 Tausch C, Konstantiniuk P, Kugler F, Reitsamer R, Roka S, Pöstlberger S, et al.; Austrian Sentinel Node Study Group. Sentinel lymph node biopsy after preoperative chemotherapy for breast cancer: findings from the Austrian Sentinel Node Study Group. Ann Surg Oncol. 2008 Dec;15(12):3378-83. 